\title{
Direct small-angle-neutron-scattering observation of stretched chain conformation in nanocomposites: More insight on polymer contributions in mechanical reinforcement
}

\author{
Nicolas Jouault, ${ }^{1}$ Florent Dalmas, ${ }^{2}$ Sylvère Said, ${ }^{3}$ Emanuela Di Cola, ${ }^{4}$ Ralf Schweins, ${ }^{5}$ Jacques Jestin, ${ }^{1, *}$ and François Boué ${ }^{1}$ \\ ${ }^{1}$ Laboratoire Léon Brillouin, CEA Saclay, 91191 Gif-Sur-Yvette, France \\ ${ }^{2}$ Institut de Chimie et des Matériaux Paris-Est, UMR 7182, CNRS, 2-8 rue Henri Dunant, 94320 Thiais, France \\ ${ }^{3}$ LIMATB, UBS, rue Saint Maudé, BP 92116, 56321 Lorient Cedex, France \\ ${ }^{4}$ ESRF, BP 220, F-38043 Grenoble Cedex, France \\ ${ }^{5}$ Institut Laue-Langevin, DS/LSS, 6 rue Jules Horowitz, BP 156, 38042 Grenoble Cedex 9, France
}

(Received 30 March 2010; published 1 September 2010)

\begin{abstract}
In this paper we present a direct measurement of stretched chain conformation in polymer nanocomposites in a large range of deformation using a specific contrast-matched small angle neutron scatttering (SANS) method. Whatever are the filler structure and the chain length the results show a clear identity of chain deformation in pure and reinforced polymer and offer more insight on the polymer chain contribution in the mechanical reinforcement. It suggests that glassy layer or glassy paths, recently proposed, should involve only a small fraction of chains. As a result, the remaining filler contribution appears strikingly constant with deformation as explained by continuous locking-unlocking rearrangement process of the particles.
\end{abstract}

DOI: 10.1103/PhysRevE.82.031801

PACS number(s): 82.35.Lr, 81.05.Qk, 61.05.fm

Polymers filled with nanoparticles (PNCs) exhibit improved mechanical properties compared to the pure matrix making them interesting materials for many applications [1]. Reinforcement mechanisms can be described according to two main contributions: one associated to the filler and the other to the chains. We focus here on chains, more specifically on their deformation, how it is affected by fillers, and the impact of this deformation on the elastic modulus. One increasingly recognized point is filler dispersion. It acts on geometrical confinement of chains, as measured [2-4] and modeled $[5,6]$ in the isotropic state, and on their interfacial dynamics: authors detected glassy polymer layers around fillers that increase the volume of the rigid phase $[7,8]$, for sufficient dispersion. Liquid layers have also been proposed, by analogy with thin films, for systems showing a decrease in the average glass transition temperature $T_{g}$ [9]. This suggested a different filler-polymer interaction, but the difference in dispersion appeared also determining [10]. Our system, well representative in the field of PNCs, associates polystyrene (PS) and silica, as extensively studied $[4,9,10]$, in two filler structures, dispersed fractal aggregates, and connected filler network. Its mechanical tests approach the most active type of nowadays industrial effort along the line of, e.g., composites with carbon black.

Up to date, the evolution of chain conformation under stretching in nanocomposites has been investigated mostly by indirect measurements. Deuterium NMR experiments $[7,8,11,12]$ on elastomers provide an average of local strain within the matrix, and deformation inhomogeneities have been observed, increasing with filler fraction, directly correlated with the increasing of mechanical reinforcement. Recent simulations yield a similar picture [13]. Getting the single chain form factor by small angle neutron scattering (SANS) $[14,15]$, homogeneous overstrain has been established in triblock copolymer micelles of PI-PS-PI in cross

\footnotetext{
*jacques.jestin@cea.fr
}

linked polyisoprene (PI) matrix, nicely dispersed but different from classical PNCs, where a direct measurement of polymer chain deformation is not available. Hence, it remains difficult to separate the chain from the filler contributions to the final macroscopic reinforcement. The present paper addresses such direct measurement.

The zero average contrast (ZAC) method, based on a mixture of nondeuterated (H) and deuterated (D) PS chains, is the only technique which enables a direct measurement inside a composite without further approximations. Then stress measurements will allows us to correlate the local chain deformation effects with influence of strain on the macroscopic material modulus and discriminate the contribution of the chain deformation from the one of the filler structure.

We have generated our model system by dispersing nanosilica $(R \approx 6 \mathrm{~nm})$ in a PS matrix. The nanocomposites were prepared by mixing a silica solution with a PS solution and drying to get three final silica volume fractions: $0 \%, 5 \%$ and $15 \% \mathrm{v} / \mathrm{v}$, characterized by a combination of $\mathrm{x}$ rays and transmission electronic microscopy [16]. Nanosilica arranges at low silica volume fraction in small aggregates, not visibly connected, with an essentially narrow size distribution, and forms a clearly connected network with mesh size of $125 \mathrm{~nm}$ for $15 \% \mathrm{v} / \mathrm{v}$. Differential scanning calorimetry gives $T_{g}$ $=94{ }^{\circ} \mathrm{C}$ for $0 \%, 95{ }^{\circ} \mathrm{C}$ for $5 \%$, and $98{ }^{\circ} \mathrm{C}$ for $15 \% \mathrm{v} / \mathrm{v}[16]$, suggesting glassy layers in the $15 \% \mathrm{v} / \mathrm{v}$ connected structure. To correct for $T_{g}$ shift effect on stress, nanocomposites of length $L$ were stretched at constant temperature $T-T_{g}$ $=20{ }^{\circ} \mathrm{C}$ with a constant rate of deformation $\epsilon=5 \times 10^{-3} \mathrm{~s}^{-1}$ up to the maximum elongation ratio $\lambda=L(t) / L(0)=1-6$, and subsequently quenched for SANS recording. The effect of filler dispersion on mechanical properties of these samples has been already detailed [16] for low and large deformations. We focus first on SANS measurements at large elongation ratios $\lambda$.

We have adapted the ZAC method to our system. It consists of making the sample by mixing $\mathrm{H}$ and D PS chains of close molecular weights in specific proportions with a third 
continuous medium, which is usually a solvent replaced with the silica in our case. The total scattering function of the resulting three-component system can be expressed by the relation

$$
\begin{aligned}
I(q)= & \left(\rho_{D}-\rho_{H}\right)^{2} x(1-x) \nu \Phi N P(q) \\
& +\left[x \rho_{D}+(1-x) \rho_{H}-\rho_{0}\right]^{2}\left[\nu \Phi N P(q)+V \Phi^{2} S(q)\right],
\end{aligned}
$$

where $\rho_{H}, \rho_{D}$, and $\rho_{0}$ are, respectively, the scattering length densities of the $\mathrm{H}$ chains, of $\mathrm{D}$ chains, and of the continuous medium (here, silica); $x$ is the molar fraction of deuterated chains; $\nu$ is the molar volume of the chain segment $\mathrm{H}$ as well as $\mathrm{D}, V$ is the total volume; and $\Phi$ is the total volume fraction of polymer in the system. $P(Q)$ and $S(Q)$ are, respectively, the form factor and the structure factor of the interchain $\mathrm{H}$ or $\mathrm{D}$. When the average scattering length density of an $\mathrm{H}$ chain/D chain mixture $(60.9 \% \mathrm{v} / \mathrm{v} / 39.1 \% \mathrm{v} / \mathrm{v})$ is equal to the one of the silica, i.e., when $\left[x \rho_{D}+(1-x) \rho_{H}-\rho_{0}\right]=0$, all correlations involving silica are cancelled out, as well as interchain correlations if the $\mathrm{H}$ and $\mathrm{D}$ chains have the same weight distribution (with polydispersity index lower than 1.2). Then only the intrachain signal, proportional to the average form factor of a single chain $P(q)$, is measured [first term of Eq. (1)]. The scattering intensity $I(q)$ described by Eq. (2) is then reduced to

$$
I(q)=\left(\rho_{D}-\rho_{H}\right)^{2} x(1-x) \nu \Phi N P(q) .
$$

However, imperfect matching of the silica filler by the H/D matrix might perturb the scattering signal. To ensure perfect matching, we used conditions different from the ZAC ones: we have synthesized some statistical H/D PS chains from a mixture of $\mathrm{H}$ and $\mathrm{D}$ styrene, whose scattering length density is equal to the one of the silica and used it with a mixture of $\mathrm{H}$ chains/D chains of mass $138 \mathrm{k}$ in ZAC proportions.

Figure 1 shows the SANS results for $M_{w}=315 \mathrm{k}$ in directions parallel and perpendicular to the stretching for direct comparison of unfilled and filled polymer matrix at 5\% silica, in $\log -\log$ representation for $\lambda=2,4$, and 6 , or for $\lambda=1.28$ for comparison with a sample filled with $15 \%$ of silica. Intensity maps of the two-dimensional (2D) detector (inset) show strong anisotropy, evidencing the deformation of the chains.

At first sight, particularly striking is the unanimous identity of chain conformation with and without fillers, in both directions, for each elongation ratio. This is true at 5\% silica from $\lambda=2$ to $\lambda=6$, as shown in Figs. 1(a)-1(c). It is also true for $15 \%$ silica: the samples were much more difficult to stretch, reaching only an elongation ratio $\lambda=1.28$ [Fig. 1(d), showing a difference at the lowest $q$ 's due to crazes]. The chain deformation is unperturbed by the presence of silica contrary to previous study [14], with different characteristic sizes and filler structures in a cross linked matrix. It is not sensitive to any confinement due to filler reorganizations resulting from the deformation either for low filler content (below the percolation threshold) or for large filler content (above the percolation threshold). This effect is clearly con-
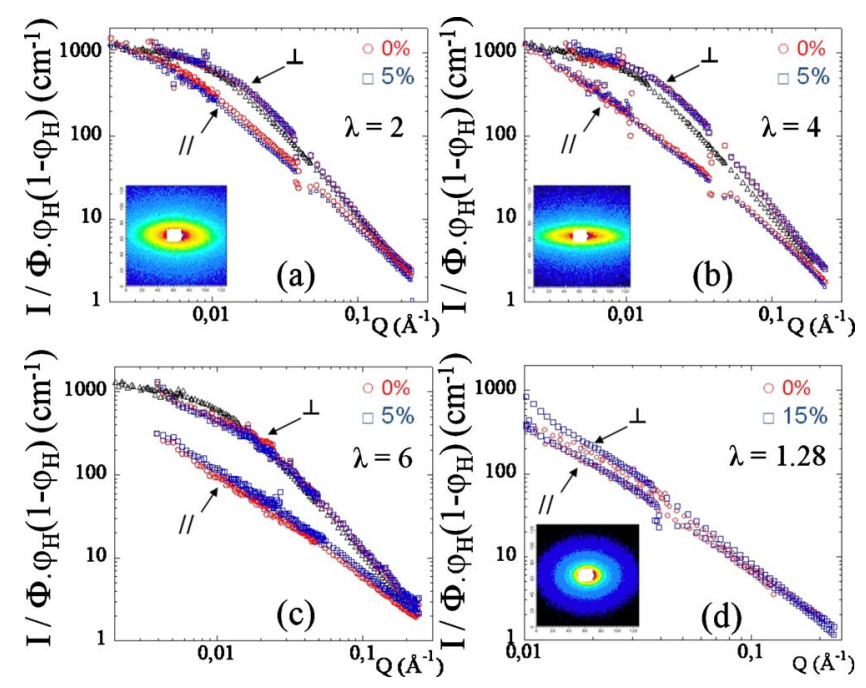

FIG. 1. (Color online) SANS results (absolute intensity) normalized by $\phi \varphi_{h}\left(1-\varphi_{h}\right)$ from chains in stretched unfilled polymer (red circle symbols) and nanocomposites filled with $5 \% \mathrm{v} / \mathrm{v}$ of silica particles (blue square symbols) for a molecular weight of $315 \mathrm{k}$ $\left(M_{w H}=315 \mathrm{~kg} / \mathrm{mol}^{-1}\right)$ in directions parallel (\|, lower symbols) and perpendicular ( $\perp$, upper symbols) to the stretching, and for elongation ratios (a) $\lambda=2$, (b) $\lambda=4$, and (c) $\lambda=6$ and (d) for higher filler content of $15 \% \mathrm{v} / \mathrm{v}$ at $\lambda=1.28$. Inset: intensity maps of the $2 \mathrm{D}$ detector. Black triangle symbols: isotropic scattering (identical in filled and unfilled unstretched samples).

firmed at 5\% in Fig. 2 for three other molecular weights: lower [138 k, Fig. 2(a)], comparable [430 k, Fig. 2(b)], and higher [1777 k, Fig. 2(c)].

Again the scattering from filled (5\%) and unfilled samples superimposes perfectly in the probed $Q$ range. The 430 and $1777 \mathrm{k}$ cases correspond to an $\mathrm{H}$ chain/D chain mixture with silica, while the $138 \mathrm{k}$ case corresponds to an $\mathrm{H}$ chain/D chain mixture in a statistical H/D matrix which matches the silica. The conclusion is the same in all cases, confirming very good matching. For the pure PS matrix, the curve is classical for stretched chains, and it has been well described in the literature. At low $Q$ (Guinier regime), the radius of gyration $R_{g}$ of the deformed chain is determined using the classical Debye function in parallel and perpendicular directions. Its experimental variation with $\lambda$ is compared (Fig. 3) with predictions for a chain inside a tube [extending the phantom network model by replacing cross links with entanglements (sliplinks)]; $R_{g}$ is calculated [17] for a chain passing by a number $k$ of sliplinks, $k=M_{w} / M_{e}$, where the mass $M_{e}$ between entanglements is 18000 for PS. For both molecular weights, the radius is less deformed, as due to chain reptation $[18,19]$ during stretching, which is faster for the lower $M_{w}$, as expected. When increasing $q$, i.e., decreasing the distance, the form factor reflects also this relaxation by a loss of anisotropy. It is known that this can be accounted by implying relaxation between the sliplinks, i.e., inside the tube, followed by reptation out of the tube $[20,21]$. The presence of the filler is expected to modify this behavior for all chains or at least a fraction of them, by creating specific polymer/filler interaction which could apply at short or long range, as a function of the filler structure. This should affect 

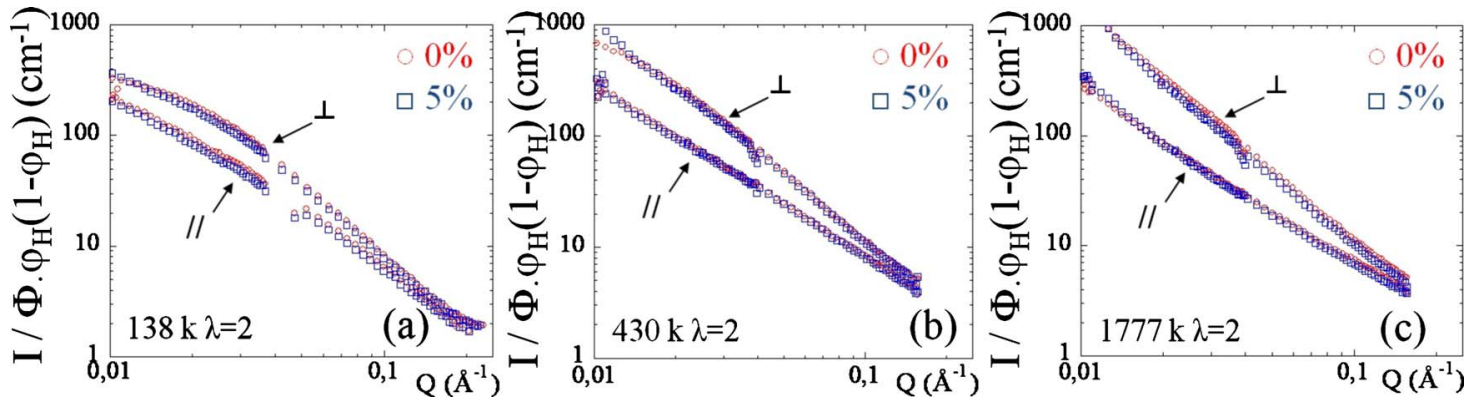

FIG. 2. (Color online) Normalized scattering of stretched unfilled polymer (red circle symbols) and 5\% v/v filled nanocomposites (blue square symbols) $\lambda=2$ for molecular weights (a) $M_{w}=138$, (b) 430 , and (c) $1777 \mathrm{k}$.

the relaxation processes (including reptation) of the chains under stretching, but such modification is clearly not observed here.

The second important result concerns the mechanical response: at variance with chain conformation, the mechanical response of the nanocomposites is very different in the pure matrix and in the reinforced polymer. For instance, for $\Phi_{\text {silica }}=15 \%$ at $\lambda=1.28$ only, the stress is larger than the matrix one by an order of magnitude [16]. For the samples observed here $(5 \% \mathrm{v} / \mathrm{v})$, the difference in stress survives at high elongation ratio as highlighted in Fig. 4(a). At this stage, it is worth noting that the curves look as shifted, with respect to the pure polymer curve, by a constant value, increasing with silica fraction. This prompts us to subtract the pure polymer curve from the composite one, in agreement also with the independence of average chain deformation on filler concentration. The remaining stress after such subtraction is presented for $315 \mathrm{k}$ in Fig. 4(a) (black triangle symbols) and for all molecular weights in Fig. 4(b). We obtain a second striking behavior: the remaining contribution to stress is quasiconstant with $\lambda$; this is seen for all polymer sizes and has also been observed for two other deformation gradients 0.001 and $0.01 \mathrm{~s}^{-1}$ (not shown).

Thanks to a well-defined model nanocomposite, we have shown that the deformation of the chains is not affected by the presence of the structured silica filler inside the nanocomposite (Figs. 1 and 2). This result suggests that the chain deformation does not contribute more than for the intrinsic

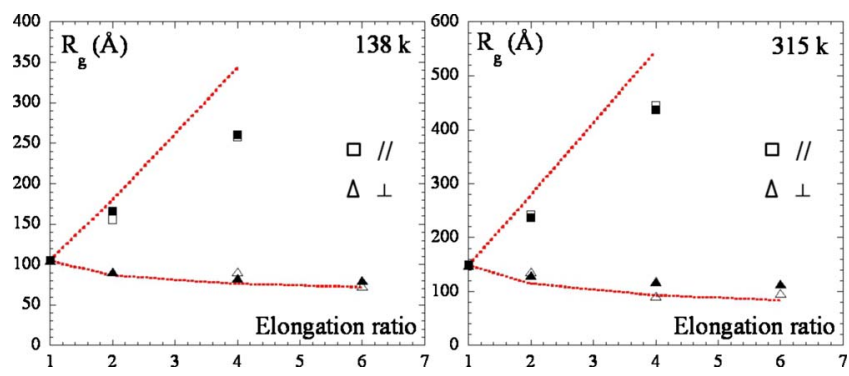

FIG. 3. (Color online) Radius of gyration of chains of molecular weights $138 \mathrm{k}$ (left) and $315 \mathrm{k}$ (right), in direction parallel (\|, squares) and perpendicular ( $\perp$, triangles) to stretching: experimental result using Debye function for filled (5\% v/v, full symbols) and unfilled ( $0 \% \mathrm{v} / \mathrm{v}$, empty symbols) polymers, and phantom network predictions (dashed red line) assuming one sliplink, each with 180 segments. matrix to the elastic modulus of the material, whatever are the silica content, the filler structure, and the polymer molecular weight. This has two important consequences with respect to the different reinforcement models. Models $[22,23]$ implying that some chains would connect elastically the fillers predict a stronger deformation of these chains: the absence of such effects suggests a very small fraction of them. A more recent approach $[8,9,24]$ proposes the existence of a fraction of glassy chains (glassy layer or glassy paths) to explain reinforcement depending on $T-T_{g}$ [25]. In a simplistic picture, glassy chains then would not be largely deformed, with the other chains undergoing most of the deformation: this should also result in a difference in average chain deformation. To enhance this difference, since glassy effects increase closer to $T_{g}$, we decreased the stretching temperature down to $T_{g}+10^{\circ} \mathrm{C}$. We see in Figs. 4(c) and 4(d) that again the scattering signal of the filled sample su-
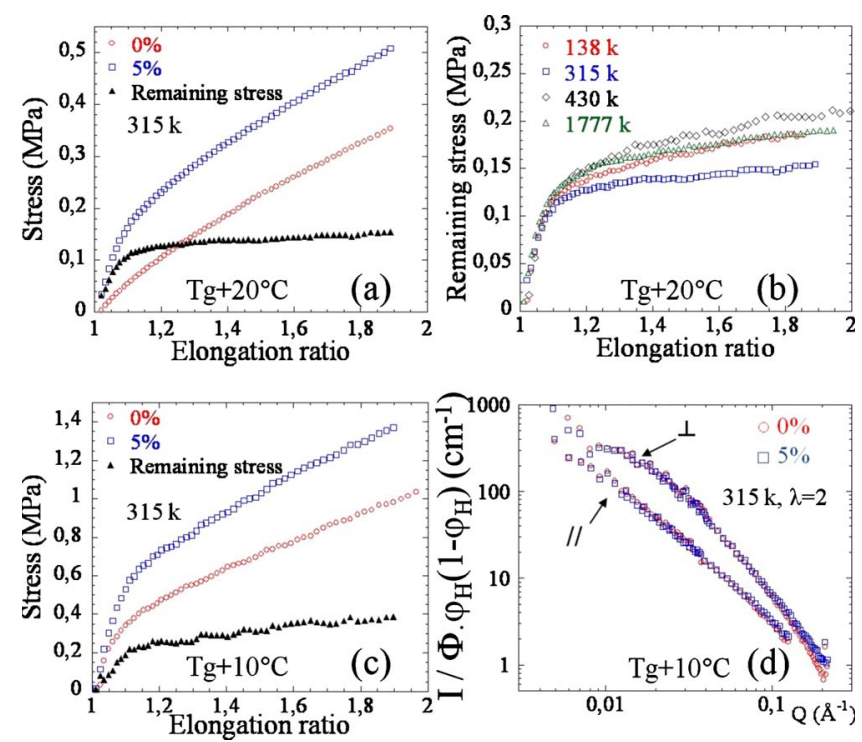

FIG. 4. (Color online) (a) Stress-strain for unfilled polymer (red circles) and 5\% filled nanocomposite (blue squares) for $M_{w}=315 \mathrm{k}$. The remaining stress after subtraction of unfilled polymer stress (black triangles) reaches a plateau above $\lambda=1$.1. (b) Remaining stress versus elongation ratio for four molecular weights (from 138 to $1777 \mathrm{k}$ ) at the same silica volume fraction of $5 \% \mathrm{v} / \mathrm{v}$. (c) and (d) Stretching closer to $T_{g}\left(T_{g}+10^{\circ} \mathrm{C}\right)$ : (c) stress-strain curves and (d) scattering intensities filled (blue squares) and unfilled (red circles) at $\lambda=2$ for $M_{w}=315 \mathrm{k}$. 
perimposes perfectly with the unfilled one, and the remaining stress is still quasiconstant with $\lambda$. To summarize, glassy effect is not excluded but clearly concerns in our case a very small fraction of chains, although our system displays a positive $T_{g}$ shift for $15 \% \mathrm{v} / \mathrm{v}$ of silica. As a result, the constant value of the remaining modulus when increasing deformation could be attributed mostly to the contribution of the filler structure. This surprising and rarely reported effect [26] could be explained by a combination of filler displacement
[27] and filler orientation [28] along the stretching direction inducing a continuous locking-unlocking process of filler associations. These observations offer more insight on the physics of chain confinement, including the actively discussed case of glass transition in ultrathin films [29], in the basic mechanisms of reinforcement, and can enable the design of new hybrid materials with improved mechanical properties in a large range of deformation.
[1] S. Tjong, Mater. Sci. Eng. R. 53, 73 (2006).

[2] A. I. Nakatani, W. Chen, R. G. Schmidt, G. V. Gordon, and C. C. Han, Polymer 42, 3713 (2001).

[3] A. Tuteja, P. M. Duxbury, and M. E. Mackay, Phys. Rev. Lett. 100, 077801 (2008).

[4] S. Sen, Y. Xie, S. K. Kumar, H. Yang, A. Bansal, D. L. Ho, L. Hall, J. B. Hooper, and K. S. Schweizer, Phys. Rev. Lett. 98, 128302 (2007).

[5] J. E. Mark, R. Abou-Hussein, T. Z. Sen, and A. Kloczkowski, Polymer 46, 8894 (2005).

[6] G. Allegra, G. Raos, and M. Vacatello, Prog. Polym. Sci. 33, 683 (2008).

[7] N. K. Dutta, N. R. Choudhury, B. Haidar, A. Vidal, J. B. Donnet, L. Delmotte, and J. M. Chazeau, Polymer 35, 4293 (1994).

[8] J. Berriot, H. Montes, F. Lequeux, D. Long, and P. Sotta, Macromolecules 35, 9756 (2002).

[9] A. Bansal, H. Yang, C. Li, K. Cho, B. C. Benicewicz, S. K. Kumar, and L. S. Schadler, Nature Mater. 4, 693 (2005).

[10] S. Sen, Y. Xie, A. Bansal, H. Yang, K. Cho, L. S. Schadler, and S. K. Kumar, Eur. Phys. J. Spec. Top. 141, 161 (2007).

[11] P. Ekanayake, H. Menge, H. Schneider, M. E. Ries, M. G. Brereton, and P. G. Klein, Macromolecules 33, 1807 (2000).

[12] S. Dupres, D. Long, P. A. Albouy, and P. Sotta, Macromolecules 42, 2634 (2009).

[13] D. Long and P. Sotta, Macromolecules 39, 6282 (2006).

[14] S. Westermann, M. Kreitschmann, W. Pyckhout-Hintzen, D. Richter, E. Straube, B. Farago, and G. Goerigk, Macromol- ecules 32, 5793 (1999).

[15] E. Straube, V. Urban, W. Pyckhout-Hintzen, D. Richter, and C. J. Glinka, Phys. Rev. Lett. 74, 4464 (1995).

[16] N. Jouault, P. Vallat, F. Dalmas, S. Said, J. Jestin, and F. Boué, Macromolecules 42, 2031 (2009).

[17] R. Ullman, Macromolecules 15, 1395 (1982).

[18] P. G. de Gennes, Scaling concepts in polymer physics (Cornell University Press, Ithaca, NY, 1999).

[19] M. Doi and S. F. Edwards, The theory of polymer dynamics (Clarendon Press, Oxford, 1986).

[20] F. Boué, Adv. Polym. Sci. 82, 47 (1997).

[21] F. Boué, J. Bastide, M. Buzier, A. Lapp, J. Herz, and T. Vilgis, Colloid Polym. Sci. 269, 195 (1991).

[22] P. G. Maier and D. Goritz, Kautsch. Gummi Kunstst. 49, 18 (1996).

[23] A. S. Sarvestani, Eur. Polym. J. 44, 263 (2008).

[24] D. Long and F. Lequeux, Eur. Phys. J. E 4, 371 (2001).

[25] J. Berriot, H. Montes, F. Lequeux, D. Long, and P. Sotta, EPL 64, 50 (2003).

[26] A. J. Meuler, G. Fleury, M. A. Hillmyer, and F. S. Bates, Macromolecules 41, 5809 (2008).

[27] J. Oberdisse, Y. Rharbi, and F. Boué, Comput. Theor. Polym. Sci. 10, 207 (2000).

[28] J. Jestin, F. Cousin, I. Dubois, C. Ménager, R. Schweins, J. Oberdisse, and F. Boué, Adv. Mater. 20, 2533 (2008).

[29] M. Alcoutlabi and G. B. McKenna, J. Phys.: Condens. Matter 17, R461 (2005). 